\title{
Revisiting Connectivity Map from a gene co-expression network analysis
}

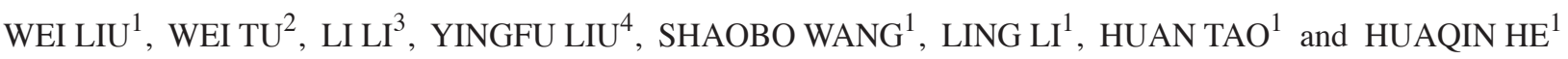 \\ ${ }^{1}$ Department of Bioinformatics, School of Life Sciences, Fujian Agriculture and Forestry University, \\ Fuzhou, Fujian 350002, P.R. China; ${ }^{2}$ Department of Molecular and Cellular Medicine, \\ Texas A\&M Health Science Center, College Station, TX 77843-1114, USA; ${ }^{3}$ Department of Medical Informatics, \\ Institute of Health Service and Medical Information, Academy of Military Medical Sciences, Beijing 100850; \\ ${ }^{4}$ Department of Cell Biology, Logistics University of Chinese Armed Police Forces, Tianjin 300309, P.R. China
}

Received October 11, 2017; Accepted December 8, 2017

DOI: $10.3892 /$ etm.2018.6275

\begin{abstract}
The Connectivity Map (CMap) is a tool that has been extensively utilized to study drug repositioning and side-effect prediction. However, most of these analyses rely on signature genes, ignoring the pathways by which those genes are regulated, as well as the functional overlap of redundant genes. The present study utilized a systems biology approach referred to as Weighted Gene Co-expression Network Analysis (WGCNA) to dissect the transcriptional profiles of CMap and reveal these hidden factors. Seven common modules associated with protein binding, extracellular matrix organization and translation were identified. Furthermore, drugs were clustered based on module expression to infer their mechanism of action (MoA) based on common activity profiles. As an extension of this, an example of disease-based module projection to identify novel drugs was provided. The analysis developed in the present study may provide a novel framework for drug repositioning or discovering MoAs.
\end{abstract}

\section{Introduction}

With the development of high-throughput drug screening, a tremendous amount of genome-wide drug interaction data has been generated. Although valid, most of this information is of lesser value to drug discovery. Due to the sheer immensity of these results, computational approaches are required to parse this data, and discover underlying patterns and networks of activity. Chemical similarities between drug and ligand sets often predict thousands of unanticipated

Correspondence to: Professor Huaqin He, Department of Bioinformatics, School of Life Sciences, Fujian Agriculture and Forestry University, 15 Shangxiadian Road, Fuzhou, Fujian 350002, P.R. China

E-mail: hehq3@fafu.edu.cn

Key words: Connectivity Map, weighted gene co-expression network analysis, drug repositioning, mechanism of action associations, suggesting that a drug may have more than one target, unintentional side effects, or making it a candidate for drug repositioning (1). Having multiple targets is not uncommon: Numerous approved drugs appear to work by modulating multiple genes, including unknown genes (2). In the clinic, numerous mono-therapies have been demonstrated to have limited effects due to gene function redundancy (3). As such, network pharmacology, the study of drug effects on protein-protein interactions, represents the next paradigm in drug discovery (4).

One of the largest pharmacological databases is provided by the Broad Institute of the Massachusetts Institute of Technology and Harvard-the Connectivity Map (CMap), comprising genome-wide gene expression profiles (from microarrays) for three human cell lines in response to cule treatments $(5,6)$. However, the reductionist methods in pharmacology focus only on the tip of an iceberg. Therefore, there is a requirement to re-analyze genome-wide pharmacological data with approaches from systems or networks (7).

Numerous studies have mined CMap to identify potential drugs with a desired action via 'signature genes' (8). For instance, Functional Module CMap used multiple functional gene modules to query the CMap and screen candidate drugs (9). The CMap tool has been used to retrieve candidate drugs via signature genes and a rank-based pattern-matching strategy (10). Iskar et al (11) identified conservative transcriptional modules between human cell lines and rat liver by analyzing CMap and DrugMatrix. Raghavan et al (12) performed a search of the CMap database to identify novel drugs for ovarian cancer based on recurrent gene signatures. Cheng et al (13) compared three CMap-based methods regarding their prediction performance against a curated dataset of 890 true drug-indication pairs and identified that XSum, which is also based on disease signatures, performs best. Thus, the premise of most CMap studies is that disease may be characterized by certain important signature genes. However, many studies have relied on its accuracy and predictive value to an unreasonable extent. First, the parameters for 'signature genes' may be influenced by population size, the cell line being studied, disease severity or differential gene expression methods (14). Furthermore, gene expression 
signatures are defined as an alteration in the expression of a gene (or genes) with validated specificity in terms of diagnosis, prognosis or prediction of therapeutic response, ignoring the possibility of gene interactions (15). The definition just focuses on the quantitative gene expression alteration, but ignores the interconnections between genes. Signature genes may be expressed with high correlation. Finally, gene function is not considered by a 'signature genes' approach, due to the possibility of redundant gene activity (16). These issues may be resolved by network biology methods, which consider not only the expression but also the modular function of genes.

To address these issues, the present study applied a method termed Weighted Gene Co-expression Network Analysis (WGCNA) to CMap and identified co-expressed gene sets (modules) with functional annotations. Although the CMap has been extensively analyzed, the common traits among these drugs are often neglected. The present analysis revealed common targets for chemical drugs among transcriptional networks, and is intended to serve as an outline for a module-based method for drug repositioning studies.

\section{Materials and methods}

Data acquisition. The microarray dataset was downloaded from the CMap server (https://portals.broadinstitute. org/cmap/cel_file_chunks.jsp). After excluding chips that were not from the Affymetrix HT_HG-U133A platform and disrupted files, 5,195 gene expression profiles from 3 cell lines (MCF7, breast cancer epithelial cells; PC3, human prostate cancer cells; HL60, human promyeloblasts) treated with 1,219 drug-like compounds were analyzed. All of these samples were pooled together for use in WGCNA algorithms to identify common modules (data not shown).

Microarray data analysis. Microarray data analysis was performed by using the Affymetrix Expression Console software (v1.4.1.46; Affymetrix Inc., Santa Clara, CA, USA) and the MAS5 normalization method. WGCNA was performed according to the manual (17). Signed co-expression networks were constructed on the genome-wide 22,215 probes using the WGCNA package (v1.51; R Foundation for Statistical Computing, Vienna, Austria; available from https://cran.r-project.org/src/contrib/WGCNA_1.51.tar.gz) (17) in R (v3.2; R Foundation for Statistical Computing; available from https://www.R-project.org) with the following parameters: Power, 6; minModuleSize, 30; deepSplit, 4; neworkType, 'signed'. In brief, for each pair of probes, a Pearson correlation coefficient matrix was calculated and the adjacency matrix was then computed by raising the correlation matrix to the power of $\beta$, [connection strength $\left.=(0.5+0.5 \times \text { correlation })^{\beta}\right]$ (17). The power of 6 was chosen using the scale-free topology criterion. The weighted network was converted into a network of topological overlap (TO)-an advanced co-expression measurement that considers not only the correlation of two probes with each other, but also the extent of their shared correlations across the weighted network (17). Probes were hierarchically clustered on the basis of their distance as measured by 1-TO. Finally, modules were identified on the dendrogram using the Dynamic Tree Cut algorithm with a height cutoff at 0.995 (18). Highly similar modules were identified by clustering and merged together. Each module was summarized using singular value decomposition so that each module eigengene (ME) represented the first principal component of the module's expression profiles (17). Thus, the ME explains the maximum amount of variation of expression levels within a module, and is considered the most representative gene expression in a module. The $\mathrm{ME}$ value was used for downstream analysis.

Functional annotation of the modules. Gene ontology (GO) enrichment analyses for identified modules were performed in the Database for Annotation, Visualization and Integrated Discovery (DAVID) (19).

Clustering of compounds. Based on the ME value, the Euclidean distance was used to cluster compounds. The hierarchical clustering was performed by average linkage clustering. The heatmap was plotted using Genesis software (v1.7.5; Thallinger Lab, Graz, Austria; available from https://genome. tugraz.at/genesisclient/genesisclient_documentation.shtml).

Drug repositioning. The CMap was projected to a nonalcoholic steatohepatitis (NASH) dataset, which was downloaded from the ArrayExpress database (accession no. E-MEXP-3291; available from www.ebi.ac.uk/arrayexpress) and resulted in an ME matrix. The compound ME value was ranked in sequential order. In general, the drug with the highest or lowest ME may be efficient against the selected disease.

Statistical analysis. The correlation between the original module and the sampled module data was calculated by 'cor' function in R. In DAVID, the overrepresentation of a term is defined as a modified Fisher's exact P-value with an adjustment for multiple tests using the Benjamini-Hochberg method.

\section{Results}

A gene co-expression network for CMap reveals common features of drug pathways. The first task of the present study was to organize and compartmentalize a selection from the CMap database into distinct activity profiles, or modules, which represent widespread gene activation as a result of exposure to drugs. The CMap gene expression profiles were reanalyzed using the WGCNA algorithm. To obtain a scale-free network, a power of 6 was chosen (Fig. 1A). Seven gene co-expression modules were identified (Fig. 1B), and the stability of these modules was verified by sampling a random selection of half of the data in each module 1,000 times and comparing it to the whole module to verify that interconnected gene activities are truly linked-an approach which has been performed previously (20). Each module's stability was expressed as the intra-module connectivity correlation (on a scale from 0 to 1 ) between the original one and the half-sampled one (Fig. 2A). The module green was the least stable module. The module significance was calculated for each module as the average gene significance of the genes within the module. Four modules had a significant association with drug concentration (Fig. 2B).

To characterize these modules, GO enrichment analysis in the following categories was performed: Biological Process, Cellular Component and Molecular Function. Modules associated with these terms (each assigned a color for simplicity 


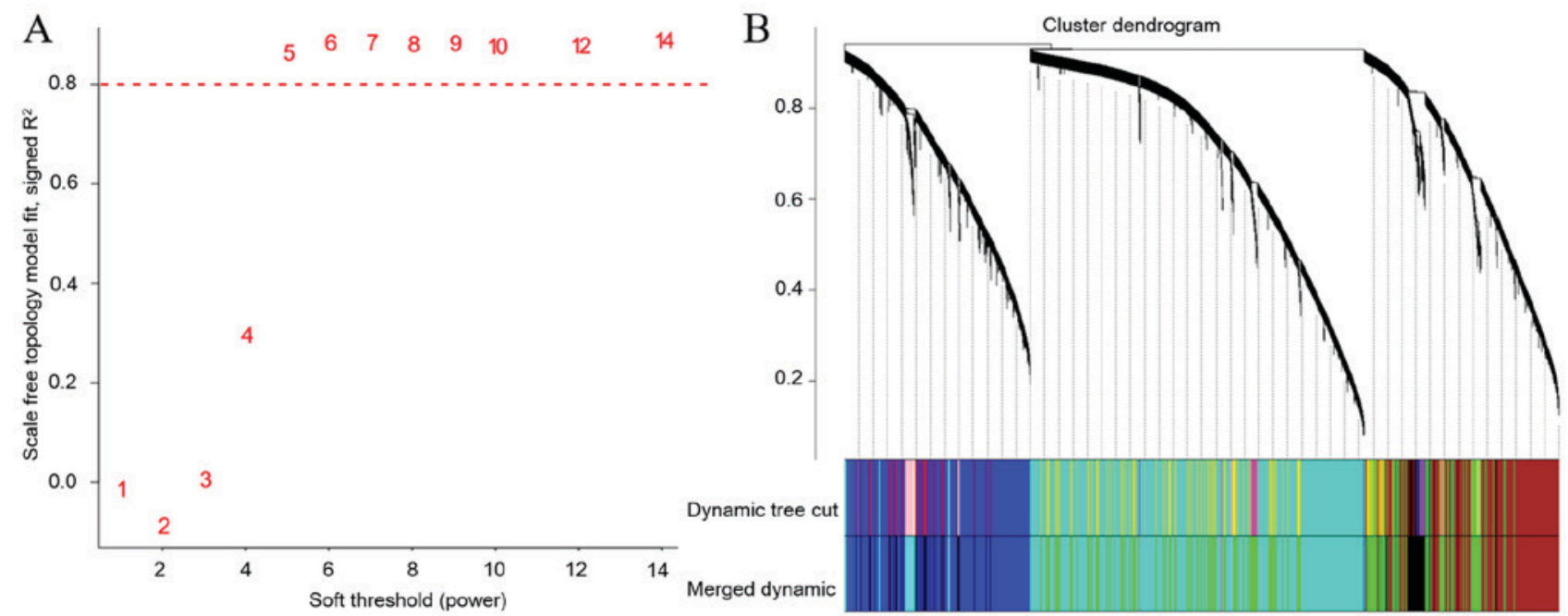

Figure 1. Seven gene co-expression modules were identified in a co-expression network for Connectivity Map. (A) Scale free topology fit ( $\mathrm{R}^{2}$, $\mathrm{y}$-axis) as a function of different powers (powers are numbers in red). The power of six was selected to construct the scale-free network using 0.8 as the cutoff. (B) Gene dendrograms displaying the co-expression modules were identified by Weighted Gene Co-expression Network Analysis and labeled by different colors. The seven module colors are blue, black, brown, white, green, grey60 and turquoise.

A

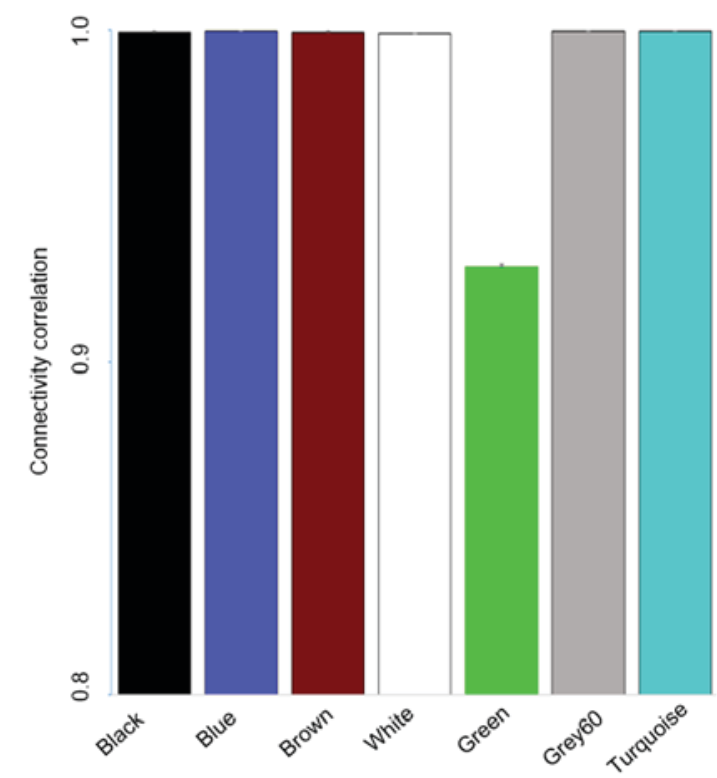

$\mathrm{B}$

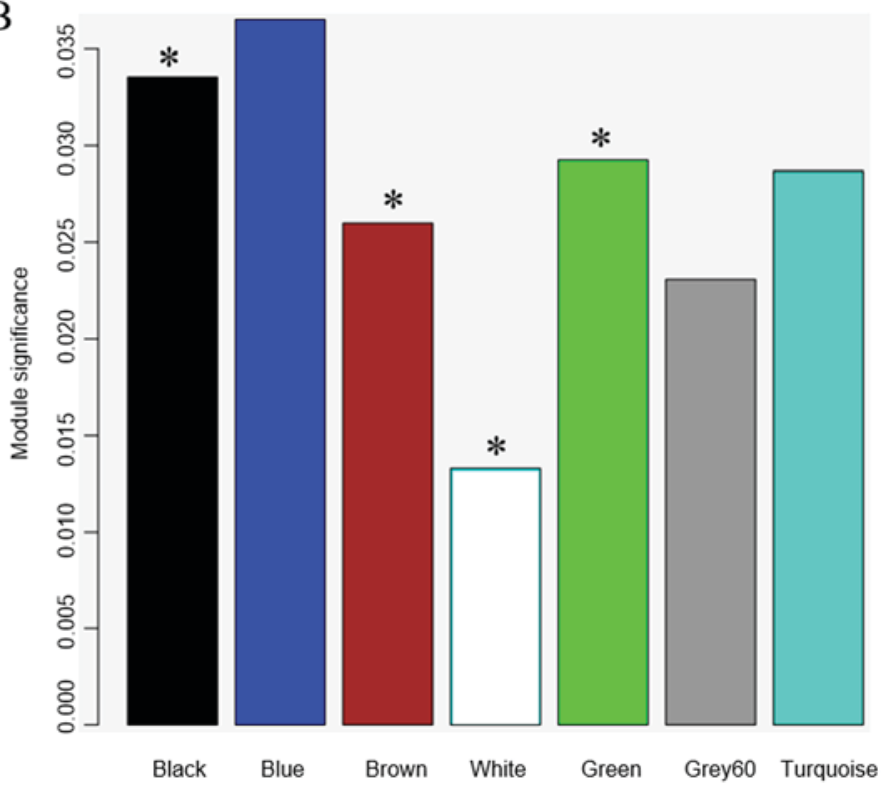

Figure 2. The identified modules were tested for their stability and significance. (A) Module stability was tested by sampling a random half of all samples 1,000 times and connectivity was correlated and expressed as the mean \pm standard deviation. (B) Module significance was also calculated by the Module significance function in WGCNA. Gene significance is the correlation of a gene expression profile with a sample trait. Module significance is determined as the average absolute gene significance measured for all genes in a given module. ${ }^{*} \mathrm{P}<0.05$ denotes the significant association of the modules with the drug concentration.

in visual mapping and naming: blue, black, brown, white, green, grey60 and turquoise) were linked to a limited range of molecular functions, which mainly comprised protein, RNA and ATP binding (Table I). Overall, modules that matched these keywords were associated with cell adhesion, extracellular matrix organization, mRNA splicing and translational initiation. Two transcriptionally distinct modules, the blue and black modules, were significantly overrepresentative of cell-cell adhesion genes, although the blue module was highly enriched in nucleoplasm protein-encoding genes and the black module was more enriched with cytosolic genes. Of note, these results suggested that most drugs examined within these modules act through protein binding.

Furthermore, samples were clustered based on ME, and three distinct clusters corresponding to three cell lines used were identified in the CMap, indicating that the cell line contributes more to gene expression than drug treatment (Fig. 3).

Computational drug clustering suggests similar mechanisms of action (MoAs). In the present study, it was hypothesized that compounds clustered together may trigger similar genes 


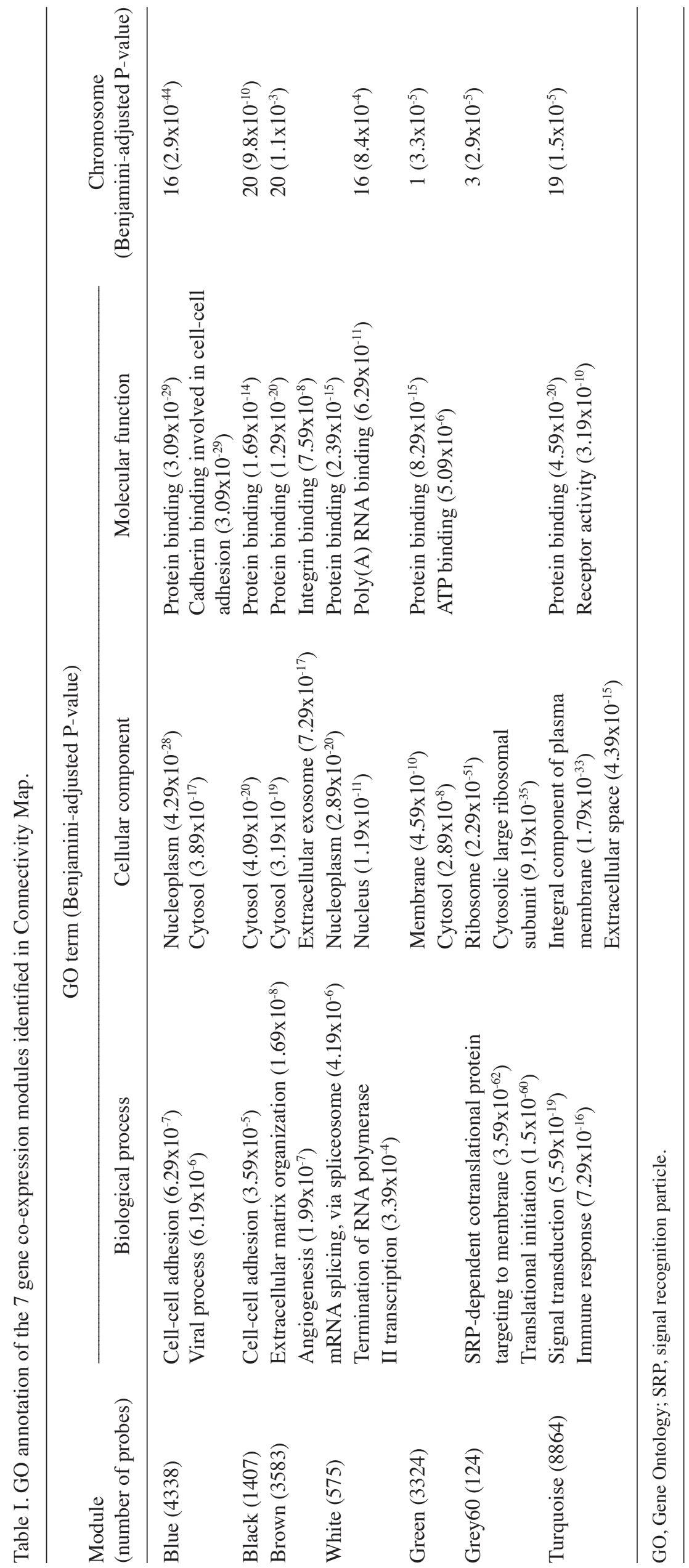




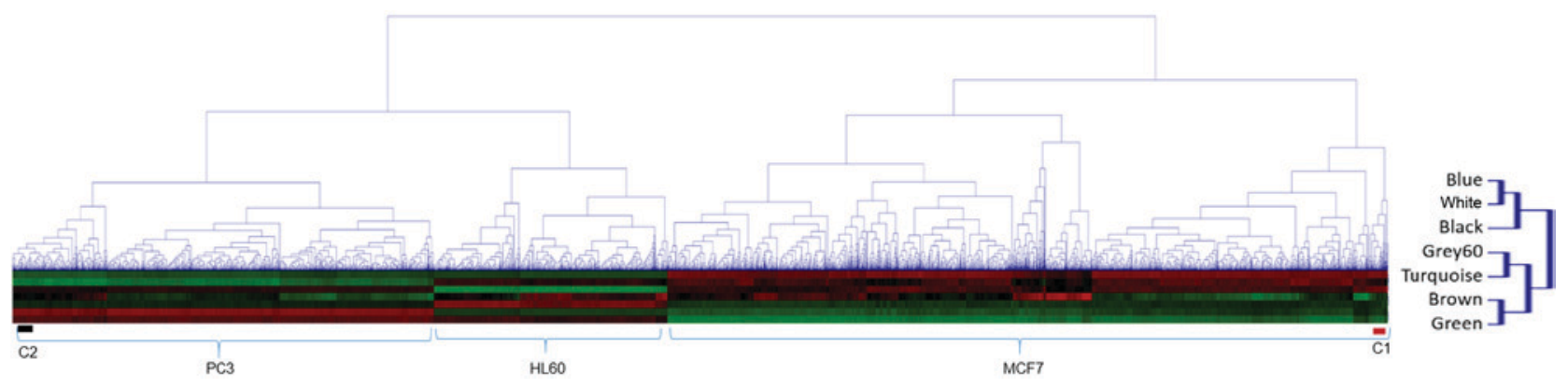

Figure 3. Connectivity Map data were hierarchically clustered based on module eigengene values. Distinct clusters corresponding to three cell lines are displayed. Two clusters, C1 (red bar) from MCF7 and C2 (black bar) from PC3, were further analyzed with the Drug-Set Enrichment Analysis tool.

and pathways, which may point to similar MoAs. If correct, a computational approach to determine a drug's MoA based on this premise may provide critical information for determining potential side-effects and repositioning. To further examine the similarity between clustered drugs by ME regarding their MoA, a recently developed computational tool, Drug-Set Enrichment Analysis (21), was used to check for common pathways shared among clustered drugs (Table II). Of note, all 50 drugs that made up Cluster 1 (C1) appeared to operate via upregulation of pathways involved in programmed cell death (data not shown). Another 54-drug cluster (C2), which was the most distant cluster from $\mathrm{C} 1$, appeared to be involved in the negative regulation of mesenchymal cell apoptotic processes $\left(\mathrm{P}=1.21 \times 10^{-4}\right)$. Several drugs from the $\mathrm{C} 2$ cluster were antibiotics (data not shown). One of the drugs from the $\mathrm{C} 2$ cluster, astemizole, has been reported to be associated with enhanced melanoma growth in mice (22), while another study suggests potential anticancer effects (23). The use of certain antibiotics has already been reported to be associated with increased risk of the incidence and fatality of breast cancer (24), and further studies should therefore be performed to ensure the safety of these drugs.

In addition, certain drugs of the $\mathrm{C} 2$ module were reported as anticancer agents (data not shown), including estradiol as a treatment for aggressive breast cancer (25) and acetylsalicylic as a preventative cancer treatment (26). However, other studies have provided contradictory reports demonstrating their carcinogenic potential; for instance, astemizole and chloramphenicol have been reported to be anticancer agents and carcinogens $(27,28)$. It is possible that some anti-cancer drugs are DNA alkylating agents and may therefore cause mutations or secondary cancers. These results, while promising, hint at the complexity in the clinically repositioning these drugs, and warrant further investigation.

Module-based analysis for drug repositioning. To provide an example for network-based drug repositioning, a previously reported network of cancer cell lines was used (20). In the present study, a module involved in the cell cycle (red module) was determined to be associated with breast cancer patient survival. The CMap microarray data were projected onto the red module, and the ME was calculated for the 1,219 drug molecules. 8-Azaguanine was identified as the top molecules for cell cycle modulation. In line with these computational predictions, 8-azaguanine has previously been reported to produce marked and reversible growth inhibition, and is clinically used as an anticancer agent $(29,30)$.
This method was also used to screen for genes associated with non-alcoholic fatty liver disease (NAFLD) (Fig. 4). A previous study by our group explored the potential drugs that may be efficient in treating NAFLD using CMap (31). The transcriptome data for NASH were used to construct a network and identify significant gene modules by WGCNA. Modules M4 and M8 were identified as significant modules. They were associated with proteasomal protein degradation (M4) and extracellular matrix (M8). The CMap data were then projected onto the NASH network, and each ME was calculated for the 1,219 chemical molecules. A total of 2 top molecules with the highest MEs were identified for M4 and M8, sulconazole and doxazosin, respectively. Of note, it has been reported that the applications of doxazosin not only include the treatment of hypertension but also the prevention of hepatic steatosis, and even the alleviation of insulin resistance (32). Doxazosin is an antihypertensives drug, but studies on its effects in NASH are currently limited to animal models (32). Sulconazole is an antifungal medication, which is used to treat skin infections (33). Its role in reducing lipid storage (patent no. US9393221; https://google.com/patents/US9393221) and anti-inflammatory (patent no. US5208015 (https://www. google.com/patents/US5208015) has been reported in two US patents. Thus, the promising roles of these drugs in NASH require verification in future clinical studies.

\section{Discussion}

CMap is a commonly used database in pharmacology, which has been utilized to identify novel drugs or MoAs. However, most recent studies focus on individual differentially expressed genes or gene signatures, ignoring the gene function redundancy and gene-gene interactions. A disease is thought to rarely be a consequence of an abnormality in a single gene, but is rather reflected by a disruption of a complex gene network (34). The present study re-analyzed the CMap microarray dataset by employing WGCNA, separating drugs and expression profiles into usable modules that may help to predict drug behaviors as well as identify MoAs. In contrast to differential expression analyses, which are based on gene signature methods, the WGCNA method analyzes whole gene clusters involved in similar biological functions. WGCNA 'modules' are more stable units than redundant individual signature genes, which may be easily influenced by screening thresholds. In addition, WGCNA may be used to take a deeper look at microarray data, revealing common mechanisms and 
Table II. The top-ranking Gene Ontology Biological Processes for clusters C1 and C2 determined with the DSEA tool.

\begin{tabular}{lrlrr}
\hline Cluster & Rank & \multicolumn{1}{c}{ Pathway name } & Escore & P-value \\
\hline C1 & 1 & Programmed cell death & 0.39 & $3.42 \times 10^{-6}$ \\
& 2 & $\begin{array}{l}\text { Positive regulation of transcription elongation } \\
\text { from RNA polymerase II promoter }\end{array}$ & 0.39 & $4.77 \times 10^{-6}$ \\
& & Viral life cycle & -0.38 & $5.65 \times 10^{-6}$ \\
C2 & 1 & Proton transport & 0.33 & $6.56 \times 10^{-5}$ \\
& 2 & Pathogen-associated molecular pattern dependent & 0.32 & $1.21 \times 10^{-4}$ \\
& & induction by symbiont of host innate immune response & & $1.45 \times 10^{-4}$ \\
\hline
\end{tabular}

DSEA, Drug-Set Enrichment Analysis; EScore, enrichment score.

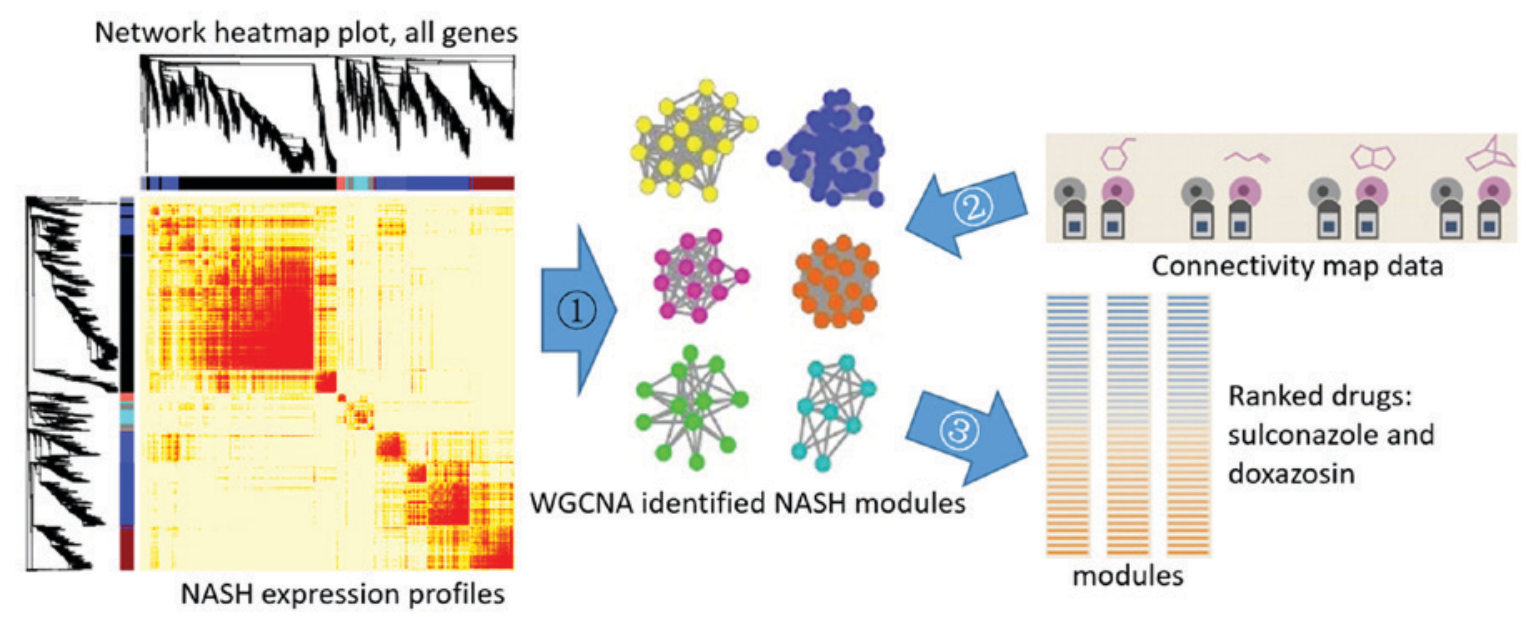

Figure 4. The rationale for disease-targeted drug repositioning was proposed. The first step is to construct gene co-expression networks for diseases of interest and to identify co-expressed modules. The second step is to project the Connectivity Map data onto these modules and calculate eigengene modules. Finally, these drugs are ranked and drug repositioning is inferred. WGCNA, Weighted Gene Co-expression Network Analysis; NASH, nonalcoholic steatohepatitis.

modes of interaction among drug sets. Of note, this analysis is based upon cellular expression profiles, not the original treatment scope of a drug, meaning that this method also provides a novel opportunity for drug repositioning.

By employing WGCNA methodology, the present study identified 7 biologically meaningful modules associated with cell-cell adhesion, extracellular matrix organization, mRNA splicing, translational initiation and signal transduction. These observed modules demonstrate the activity association of the screened drugs with each other. Clustering based on the ME value may then be performed in order to identify drugs with a similar MoA. Build upon.

Previously, network based techniques have been used for drug repositioning (35). Park et al (36) proposed a network mirroring method, assuming that if two diseases are similar, then a drug for one disease can be effective against the other disease too. Gottlieb et al (37) depended on the observation that similar drugs are indicated for similar diseases to compare multiple drug-drug and disease-disease similarity measures for drug repositioning. Chen et al (38) used bipartite network topology to prioritize the potentially indicated diseases that a drug treats. Luo et al (39) integrated diverse information, including DrugBank database, HPRD database and Comparative Toxicogenomics database, and developed a computational pipeline, called DTINet, to predict novel drug-target interactions from a constructed heterogeneous network. The limitations of these methods include the lack of negative samples and known drug-disease association information. The method in the present study only required the disease and drug gene expression profiles regardless of prior knowledge including protein-protein interactions, drug-disease associations or other sources of information.

Personalized medicine at the molecular level is a useful method to identify drugs tailored to a specific disorder (40). In theory, CMap-based techniques may be used to identify dysfunctional gene expression modules in an affected patient, guiding the clinician to identify drugs that specifically target the dysfunctional gene expression underlying that patient's disorder. Based on this premise, the present study demonstrated how a gene expression phenotype associated with cellular dysregulation may be selected (breast cancer cell cycle), how feature modules of interest associated with that phenotype may be identified and how drugs may be projected onto these modules to identify novel drugs worthy of further investigation and for application to a specific patient's condition. 
Based upon this rationale, the present study provided a novel drug discovery/repositioning system. To identify novel treatments for specific diseases or disease subtypes, WGCNA may be used to identify the dysregulated gene expression modules and cross-reference the CMap drug data to the dysregulated modules. The drugs identified may then be ranked according their ME values, and drugs may be screened simply according to their ME.

\section{Acknowledgements}

Not applicable.

\section{Funding}

This work was supported in part by the National Natural Science Foundation of China (grant nos. 31270454 and 81502091). The authors apologize that not all insightful CMap-associated studies were cited due to lack of space.

\section{Availability of data and materials}

The datasets used and/or analyzed during the current study are available from the corresponding author on reasonable request.

\section{Authors' contributions}

$\mathrm{HH}$ and HT designed the study. SW and LingL collected the data, YL and LiL analyzed the CMap and NAFLD data, WL and WT analyzed the results and drafted the manuscript.

\section{Ethics approval and consent to participate}

Not applicable.

\section{Consent for publication}

Not applicable.

\section{Competing interests}

The authors declare that they have no competing interests.

\section{References}

1. Keiser MJ, Setola V, Irwin JJ, Laggner C, Abbas AI, Hufeisen SJ, Jensen NH, Kuijer MB, Matos RC, Tran TB, et al: Predicting new molecular targets for known drugs. Nature 462: 175-181, 2009.

2. Kibble M, Saarinen N, Tang J, Wennerberg K, Mäkelä S and Aittokallio T: Network pharmacology applications to map the unexplored target space and therapeutic potential of natural products. Nat Prod Rep 32: 1249-1266, 2015.

3. Lin J, Wu L, Bai X, Xie Y, Wang A, Zhang H, Yang X, Wan X, Lu X, Sang X and Zhao H: Combination treatment including targeted therapy for advanced hepatocellular carcinoma. Oncotarget 7: 71036-71051, 2016.

4. Hopkins AL: Network pharmacology: The next paradigm in drug discovery. Nat Chem Biol 4: 682-690, 2008.

5. Lamb J, Crawford ED, Peck D, Modell JW, Blat IC, Wrobel MJ, Lerner J, Brunet JP, Subramanian A, Ross KN, et al: The Connectivity Map: Using gene-expression signatures to connect small molecules, genes, and disease. Science 313: 1929-1935, 2006

6. Qu XA and Rajpal DK: Applications of Connectivity Map in drug discovery and development. Drug Discov Today 17: 1289-1298, 2012.
7. Leung EL, Cao ZW, Jiang ZH, Zhou H and Liu L: Network-based drug discovery by integrating systems biology and computational technologies. Brief Bioinform 14: 491-505, 2013.

8. Zhang QY, Chu XY, Jiang LH, Liu MY, Mei ZL and Zhang HY: Identification of non-electrophilic Nrf2 activators from approved drugs. Molecules 22: pii: E883, 2017.

9. Chung FH, Chiang YR, Tseng AL, Sung YC, Lu J, Huang MC, Ma N and Lee HC: Functional module Connectivity Map (FMCM): A framework for searching repurposed drug compounds for systems treatment of cancer and an application to colorectal adenocarcinoma. PLoS One 9: e86299, 2014.

10. Zhou W, Ma CX, Xing YZ and Yan ZY: Identification of candidate target genes of pituitary adenomas based on the DNA microarray. Mol Med Rep 13: 2182-2186, 2016.

11. Iskar M, Zeller G, Blattmann P, Campillos M, Kuhn M, Kaminska KH, Runz H, Gavin AC, Pepperkok R, van Noort V and Bork P: Characterization of drug-induced transcriptional modules: Towards drug repositioning and functional understanding. Mol Syst Biol 9: 662, 2013.

12. Raghavan R, Hyter S, Pathak HB, Godwin AK, Konecny G, Wang C, Goode EL and Fridley BL: Drug discovery using clinical outcome-based Connectivity Mapping: Application to ovarian cancer. BMC Genomics 17: 811, 2016.

13. Cheng J, Yang L, Kumar V and Agarwal P: Systematic evaluation of Connectivity Map for disease indications. Genome Med 6: 540, 2014

14. Patil P, Bachant-Winner PO, Haibe-Kains B and Leek JT: Test set bias affects reproducibility of gene signatures. Bioinformatics 31: 2318-2323, 2015

15. Chibon F: Cancer gene expression signatures-the rise and fall? Eur J Cancer 49: 2000-2009, 2013.

16. Ivliev AE, t Hoen PA and Sergeeva MG: Coexpression network analysis identifies transcriptional modules related to proastrocytic differentiation and sprouty signaling in glioma. Cancer Res 70: 10060-10070, 2010 .

17. Zhang B and Horvath S: A general framework for weighted gene co-expression network analysis. Stat Appl Genet Mol Biol 4: Article17, 2005.

18. Langfelder P, Zhang B and Horvath S: Defining clusters from a hierarchical cluster tree: The dynamic tree cut package for $\mathrm{R}$. Bioinformatics 24: 719-720, 2008.

19. Huang da W, Sherman BT and Lempicki RA: Bioinformatics enrichment tools: Paths toward the comprehensive functional analysis of large gene lists. Nucleic Acids Res 37: 1-13, 2009.

20. Liu W, Li L and Li W: Gene co-expression analysis identifies common modules related to prognosis and drug resistance in cancer cell lines. Int J Cancer 135: 2795-2803, 2014.

21. Napolitano F, Sirci F, Carrella D and di Bernardo D: Drug-set enrichment analysis: A novel tool to investigate drug mode of action. Bioinformatics 32: 235-241, 2016.

22. Brandes LJ, Warrington RC, Arron RJ, Bogdanovic RP, Fang W, Queen GM, Stein DA, Tong J, Zaborniak CL and LaBella FS: Enhanced cancer growth in mice administered daily human-equivalent doses of some H1-antihistamines: predictive in vitro correlates. J Natl Cancer Inst 86: 770-775, 1994.

23. de Guadalupe Chávez-López M, Hernández-Gallegos E, Vázquez-Sánchez AY, Gariglio PandCamacho J: Antiproliferative and proapoptotic effects of astemizole on cervical cancer cells. Int J Gynecol Cancer 24: 824-828, 2014.

24. Velicer CM, Heckbert SR, Lampe JW, Potter JD, Robertson CA and Taplin SH: Antibiotic use in relation to the risk of breast cancer. JAMA 291: 827-835, 2004.

25. Hwang KA, Park MA, Kang NH, Yi BR, Hyun SH, Jeung EB and Choi KC: Anticancer effect of genistein on BG-1 ovarian cancer growth induced by $17 \beta$-estradiol or bisphenol A via the suppression of the crosstalk between estrogen receptor $\alpha$ and insulin-like growth factor-1 receptor signaling pathways. Toxicol Appl Pharmacol 272: 637-646, 2013.

26. Dubé C, Rostom A, Lewin G, Tsertsvadze A, Barrowman N, Code C, Sampson M and Moher D; U.S. Preventive Services Task Force: The use of aspirin for primary prevention of colorectal cancer: a systematic review prepared for the U.S. preventive services task force. Ann Intern Med 146: 365-375, 2007.

27. Brambilla G, Mattioli F, Robbiano L and Martelli A: Studies on genotoxicity and carcinogenicity of antibacterial, antiviral, antimalarial and antifungal drugs. Mutagenesis 27: 387-413, 2012. 
28. Weisburger JH, Shirasu Y, Grantham PH and Weisburger EK: Chloramphenicol, protein synthesis, and the metabolism of the carcinogen $\mathrm{N}$-2-fluorenyldiacetamide in rats. Inhibition by chloramphenicol of carcinogen binding. J Biol Chem 242: 372-378, 1967.

29. Kidder GW, Dewey VC and Parks RE Jr: Effect of lowered essential metabolites on 8-azaguanine inhibition. J Biol Chem 197: 193-198, 1952.

30. Sugiura K, Hitchings GH, Cavalieri LF and Stock CC: The effect of 8-azaguanine on the growth of carcinoma, sarcoma, osteogenic sarcoma, lymphosarcoma and melanoma in animals. Cancer Res 10: 178-185, 1950.

31. Ye $\mathrm{H}$ and Liu W: Transcriptional networks implicated in human nonalcoholic fatty liver disease. Mol Genet Genomics 290 1793-1804, 2015

32. Menacho-Márquez M, Nogueiras R, Fabbiano S, Sauzeau V, Al-Massadi O, Diéguez C and Bustelo XR: Chronic sympathoexcitation through loss of Vav3, a Rac1 activator, results in divergent effects on metabolic syndrome and obesity depending on diet. Cell Metab 18: 199-211, 2013.

33. Lassus A, Forström S and Salo O: A double-blind comparison of sulconazole nitrate $1 \%$ cream with clotrimazole $1 \%$ cream in the treatment of dermatophytoses. Brit J Dermatol 108: 195-198, 1983.

34. Barabási AL, Gulbahce N and Loscalzo J: Network medicine: A network-based approach to human disease. Nat Rev Genet 12: 56-68, 2011.
35. Wu Z, Wang Y and Chen L: Network-based drug repositioning. Mol Biosyst 9: 1268-1281, 2013.

36. Park S, Lee DG and Shin H: Network mirroring for drug repositioning. BMC Med Inform Decis Mak 17 (Suppl 1): S55, 2017.

37. Gottlieb A, Stein GY, Ruppin E and Sharan R: PREDICT: A method for inferring novel drug indications with application to personalized medicine. Mol Syst Biol 7: 496, 2011.

38. Chen H, Zhang H, Zhang Z, Cao Y and Tang W: Network-based inference methods for drug repositioning. Comput Math Methods Med 2015: 130620, 2015.

39. Luo Y, Zhao X, Zhou J, Yang J, Zhang Y, Kuang W, Peng J, Chen L and Zeng J: A network integration approach for drug-target interaction prediction and computational drug repositioning from heterogeneous information. Nat Commun 8: 573, 2017.

40. Li YY and Jones SJ: Drug repositioning for personalized medicine. Genome Med 4: 27, 2012.

(i) (3) This work is licensed under a Creative Commons Attribution-NonCommercial-NoDerivatives 4.0 International (CC BY-NC-ND 4.0) License. 